\title{
SOME FORMULAS FOR THE ENERGY FUNCTIONAL OF A MARKOV PROCESS
}

\author{
by \\ P. J. Fitzsimmons* and R. K. Getoor*
}

\section{Introduction}

In this paper we shall establish two formulas relating the energy functional of a Markov process to that of a subprocess. Let $X$ be a right Markov process and $M$ an exact multiplicative functional of $X$. Writing $(X, M)$ for the corresponding subprocess, let $L$ and $L^{M}$ denote the energy functionals of $X$ and $(X, M)$ respectively. Suppose that $M$ doesn't vanish on $\left[0, \zeta\left[\right.\right.$, and define an additive functional $A$ by $d A_{t}=-d M_{t} / M_{t-}$. Then given an $X$-excessive measure $\xi$ and an $X$-excessive function $u$ we have

$$
L^{M}(\xi, u)=L(\xi, u)+\nu^{\xi}(u)
$$

where $\nu^{\xi}$ is the Revuz measure of $A$ relative to $X$ and $\xi$. Formula (1.1) appears as (3.27) in $[\mathbf{G S t}]$ in the special case $M_{t}=e^{-q t}$.

The validity of (1.1) relies heavily on the strict positivity of $M$. Our second formula serves as a replacement for (1.1) in the general case. To state this formula we need the balayage operator $R_{M}$ associated with $M ; R_{M}$ operates on the cone of $X$-excessive

\footnotetext{
* Research supported in part by NSF Grant DMS 87-21347.
} 
measures and is the dual of the operator $P_{M}$ defined by

$$
P_{M} f(x)= \begin{cases}-P^{x} \int_{0}^{\infty} f \circ X_{t} d M_{t}, & x \in E_{M} \\ f(x), & x \in E \backslash E_{M},\end{cases}
$$

where $E_{M}=\left\{x \in E: P^{x}\left(M_{0}=1\right)=1\right\} . P_{M}$ is the analogue of the hitting operator $P_{B}$ with which it coincides if $M=1_{\left[0, T_{B}\right.}, T_{B}$ being the hitting time of $B$. This notation established we have our second formula

$$
\begin{aligned}
L(\xi, u) & =L^{M}(\tilde{\xi}, \tilde{u})+L\left(\xi, P_{M} u\right) \\
& =L^{M}(\tilde{\xi}, \tilde{u})+L\left(R_{M} \xi, u\right)
\end{aligned}
$$

where $\xi$ and $u$ are as for (1.1), $\tilde{\xi}=\xi-R_{M} \xi$, and $\tilde{u}=u-P_{M} u$. (See $\S 3$ for the precise definition of $R_{M}, \tilde{\xi}$, and $\tilde{u}$.)

Section 2 contains the proof of (1.1); this proof depends on (2.2) which is of interest in its own right. The balayage operator $R_{M}$ is discussed in $\S 3$; here we omit many details, referring the reader to $[\mathbf{F G}]$ for full proofs in the special case $M=1_{\left[0, T_{B}[\right.}$. Formula $(1.3)$ is proved in $\S 4$. The argument involves a probabilistic identification of the three terms in (1.3) and is perhaps of more interest than the formula itself.

In the remainder of this section we set down our notation and blanket hypotheses. Unexplained terminology can be found in $[\mathbf{S h}]$ or $[\mathbf{F G}]$ and the reader is advised to have a copy of the latter at hand for reference.

Throughout the paper, $X=\left(\Omega, \mathcal{F}, \mathcal{F}_{t}, X_{t}, \theta_{t}, P^{x}\right)$ is a right Markov process in the sense of $[\mathbf{S h} ; \S 20]$ with state space $(E, \mathcal{E})$, semigroup $\left(P_{t}\right)$, and resolvent $\left(U^{q}\right)$. In particular, $E$ is a separable Radon space and $\mathcal{E}$ is the $\sigma$-field of Borel sets for the Ray topology, $\left(\mathcal{F}_{t}\right)$ is the usual augmentation of the natural filtration of $X$, and conditions (20.4) and (20.5) in $[\mathbf{S h}]$ are in force. The semigroup of $X$ need only be subMarkovian, and $\zeta$ denotes the lifetime of $X$. 
Let $S^{q}(X)$ denote the cone of $q$-excessive functions of $X$ and put $\mathcal{E}^{e}=$ $\sigma\left\{\bigcup_{q \geq 0} S^{q}(X)\right\}$. As a rule the letter $f$ always denotes a positive $\mathcal{E}^{e}$-measurable function on $E$. The cone of $q$-excessive measures for $X$ is denoted $\operatorname{Exc}^{q}(X)$. As usual, when $q=0$ it vanishes from the notation; in particular $U=U^{0}$ is the potential kernel for $X$. The notational scheme for the various subcones of $\operatorname{Exc}(X)$ is as in $[\mathbf{F G}]$ and $[\mathbf{G S t}]$. Thus $\operatorname{Pur}(X)$ (resp. Inv $(X)$, Dis $(X)$, Con $(X)$ ) denotes the class of purely excessive (resp. invariant, dissipative, conservative) elements of $\operatorname{Exc}(X)$. The analogous classes over a subprocess $(X, M)$ are denoted $S(X, M)$, Exc $(X, M)$, Pur $(X, M)$, etc.

We fix once and for all an exact multiplicative functional (MF), $M$, of $X$ as specified in $[\mathbf{F G} ;(2.1)]$. As a matter of convention we assume that $M_{t}(\omega)=0$ for all $t \geq \xi(\omega)$ and $\omega \in \Omega$. Define

$$
\begin{aligned}
S & =\inf \left\{t: M_{t}=0\right\} \\
E_{M} & =\left\{x \in E: P^{x}\left(M_{0}\right)=1\right\}=\left\{x \in E: P^{x}(S>0)=1\right\} .
\end{aligned}
$$

Then $S$ is a perfect, though not necessarily exact, terminal time and $E_{M}$, the set of permanent points of $M$, is $\mathcal{E}^{e}$-measurable and serves as the state space of the subprocess $(X, M)$. We write $\left(Q_{t}\right)$ and $\left(V^{q}\right)$ for the semigroup and resolvent of $(X, M)$. For example

$$
Q_{t} f(x)=P^{x}\left(f \circ X_{t} M_{t}\right), \quad t \geq 0, x \in E .
$$

Associated with $M$ is the additive functional $(\mathrm{AF})$ of $(X, S)$

$$
d A_{t}=-1_{] 0, S[}(t) d M_{t} / M_{t-}
$$

which has Revuz measure (relative to $\xi$ )

$$
\nu^{\xi}(f):=\uparrow \lim _{t \downarrow 0} t^{-1} P^{\xi} \int_{] 0, t]} f \circ X_{s} d A_{s} .
$$


We write $U_{M}^{q}$ for the $q$-potential operator of $A$; namely,

$$
U_{M}^{q} f(x)=-P^{x}\left(\int_{] 0, S[} e^{-q t} f \circ X_{t} d M_{t} / M_{t-}\right) .
$$

The formula

$$
\nu^{\xi}(f)=\uparrow \lim _{q \rightarrow \infty} q \xi U_{M}^{q+p} f, \quad p \geq 0
$$

can be found in $[\mathbf{F G} ;(2.15)]$. Note that if $M_{t}=e^{-q t} 1_{[0, \zeta[}$, then $\nu^{\xi}=q \cdot \xi$. As noted by Meyer [M66], if $S \geq \zeta$ a.s., then

$$
U^{q}=V^{q}+U_{M}^{q} V^{q}
$$

this formula plays an important role in the sequel.

Finally, recall the energy functional $L: \operatorname{Exc}(X) \times S(X) \rightarrow[0, \infty]$ determined by

$$
L(\xi, u)=\sup \{\mu(u): \mu U \in \operatorname{Exc}(X), \mu U \leq \xi\}
$$

for $\xi \in \operatorname{Exc}(X), u \in S(X)$. The reader is referred to [DM; XII-39], [GSt], or [FG] for the various properties of the bilinear form $L$. The energy functional of $(X, M)$ is denoted $L^{M}$, and that of the $q$-subprocess $X^{q}=\left(X, e^{-q \cdot} 1_{[0, \zeta[}\right)$ is denoted $L^{q}$.

\section{Proof of (1.1).}

In this section we assume that the $\operatorname{MF}\left(M_{t}\right)$ satisfies $M_{t}>0$ for all $0 \leq t<\zeta$ a.s.; that is, the terminal time $S$ defined by (1.4) satisfies $P^{x}(S<\zeta)=0$ for all $x \in E$. In particular, $E_{M}=E$.

We begin the proof of (1.1) by noting that it suffices to consider the two special cases (i) $\xi \in \operatorname{Dis}(X)$, and (ii) $\xi \in \operatorname{Con}(X)$. This is because all three terms in (1.1) are additive in $\xi$, and since each $\xi \in \operatorname{Exc}(X)$ admits a unique decomposition $\xi=\xi_{d}+\xi_{c}$ where $\xi_{d} \in \operatorname{Dis}(X), \xi_{c} \in \operatorname{Con}(X)$. 
(i) Assume $\xi \in \operatorname{Dis}(X)$ and choose a sequence $\left(\mu_{n}\right)$ of measures on $E$ such that $\mu_{n} U \uparrow \xi$. Define $\nu_{n}=\mu_{n}+\mu_{n} U_{M}$, so that $\nu_{n} V=\mu_{n} U \uparrow \xi$ by (1.8) with $q=0$. By well-known properties of the energy functionals $L^{M}$ and $L$ (see $[\mathbf{G S t}]$ or $[\mathbf{F G}]$ ) we have

$$
\begin{aligned}
L^{M}(\xi, u) & =\uparrow \lim \nu_{n}(u)=\uparrow \lim \left[\mu_{n}(u)+\mu_{n} U_{M}(u)\right] \\
& =L(\xi, u)+\nu^{\xi}(u) .
\end{aligned}
$$

The last equality above follows from [FG; (2.18)]. (This argument is a trivial modification of that used in $[\mathbf{G S t}]$ for the special case $\left.M_{t}=e^{-q t} 1_{[0, \zeta[.}\right)$

Before proceeding with the proof of (1.1) in the case $\xi \in \operatorname{Con}(X)$ we record two facts

(2.1) LEMMA. Given $\xi \in \operatorname{Con}(X)$ let $\xi=\xi_{i}+\xi_{p}$ be the decomposition of $\xi$ into its invariant and purely excessive parts relative to $(X, M)$. Then both $\xi_{i}$ and $\xi_{p}$ lie in $\operatorname{Con}(X)$.

Proof. If $\xi(f)<\infty$ then since $\operatorname{Con}(X) \subset \operatorname{Inv}(X)$,

$$
\begin{aligned}
\xi_{p} P_{t}(f) & =\xi P_{t} f-\xi_{i} P_{t} f \leq \xi(f)-\xi_{i} Q_{t} f \\
& =\xi(f)-\xi_{i}(f)=\xi_{p}(f),
\end{aligned}
$$

and so $\xi_{p} \in \operatorname{Exc}(X)$. But then $\xi_{p} \in \operatorname{Con}(X)$ since $\xi_{p} \leq \xi \in \operatorname{Con}(X)$. Therefore, $\xi_{i}=\xi-\xi_{p}$ is seen first to lie in $\operatorname{Inv}(X)$, then in $\operatorname{Con}(X)$, being dominated by $\xi$. $\square$

Recall that $\left(V^{q}\right)$ is the resolvent of $(X, M)$ and that $V=V^{0}$.

(2.2) PROposition. (a) If $\xi \in \operatorname{Con}(X) \cap \operatorname{Inv}(X, M)$, then $\xi \in \operatorname{Con}(X, M), \nu^{\xi}=0$, and $M=1_{[0, \zeta[}$ a.s. $P^{\xi}$.

(b) If $\xi \in \operatorname{Inv}(X)$, then $\xi_{p}=\nu^{\xi} V$, where $\xi_{p}$ denotes the purely excessive part of $\xi$ relative to $(X, M)$.

Before proving (2.2) let us use it and (2.1) to finish the proof of (1.1). Thus suppose that $\xi \in \operatorname{Con}(X)$. By Lemma (2.1) we need only consider the special cases $\xi \in \operatorname{Con}(X) \cap$ $\operatorname{Inv}(X, M)$ and $\xi \in \operatorname{Con}(X) \cap \operatorname{Pur}(X, M)$. In the first case all terms in (1.1) vanish because 
of (2.2a) and [GSt; (3.11)]. In the second case $\xi=\nu^{\xi} V$ by $(2.2 \mathrm{~b})$, hence $L^{M}(\xi, u)=\nu^{\xi}(u)$ while $L(\xi, u)=0$ by the properties of $L^{M}$ and $L$; see $[\mathbf{F G}]$ or $[\mathbf{G S t}]$. $\square$

Proof of (2.2). (a) Assume $\xi \in \operatorname{Con}(X) \cap \operatorname{Inv}(X, M)$. Then by (1.8), if $q>0$,

$$
\xi=q \xi U^{q}=q \xi V^{q}+q \xi U_{M}^{q} V^{q}=\xi+q \xi U_{M}^{q} V^{q}
$$

so $\xi U_{M}^{q} V^{q}=0$ and $\xi U^{q}=\xi V^{q}$, even when $q=0$. Since $U^{q} \geq V^{q}$, the last equality yields $U^{q}(x, \cdot)=V^{q}(x, \cdot)$ for $\xi$ a.e. $x \in E$. But $\xi \in \operatorname{Con}(X)$ so if $f>0$ then $V f=U f=\infty$ a.e. $\xi$, hence $\xi \in \operatorname{Con}(X, M)$. Moreover, $U^{1} 1=V^{1} 1$ a.e. $\xi$, which implies that $M=1_{[0, \zeta[}$ a.s. $P^{\xi}$. Since $V^{q} 1>0$ (recall that $E_{M}=E$ ), $\xi U_{M}^{q} V^{q}=0$ implies $\xi U_{M}^{q}=0$, hence $\nu^{\xi}=0$ in view of (1.7).

(b) Now assume $\xi \in \operatorname{Inv}(X)$ and fix $f \in b p \mathcal{E} \cap L^{1}(\xi)$. Since $\xi \in \operatorname{Inv}(X) \subset \operatorname{Exc}(X, M)$, the first two equalities in (2.4) are valid; letting $q \rightarrow \infty$ we obtain

$$
\lim _{q \rightarrow \infty} q \xi U_{M}^{q} V^{q} f=0 .
$$

But by (1.7) and the resolvent equation for $\left(V^{q}\right)$

$$
\nu^{\xi} V f=\uparrow \lim _{p \downarrow 0} \uparrow \lim _{q \uparrow \infty} q \xi U_{M}^{q} V^{p} f=\lim _{p \downarrow 0} \lim _{q \uparrow \infty} q \xi U_{M}^{q}\left(V^{q} f+(q-p) V^{q} V^{p} f\right) .
$$

Using now (2.5), (1.8), $\xi \in \operatorname{Inv}(X)$, and the resolvent equation, the last displayed line equals

$$
\lim _{p \downarrow 0} \lim _{q \uparrow \infty} q(q-p) \xi\left(U^{q}-V^{q}\right) V^{p} f=\lim _{p \downarrow 0} \lim _{q \uparrow \infty} q \xi V^{q} f-p \xi V^{p} f=\xi_{p}(f),
$$

because $p \xi V^{p} \rightarrow \xi_{i}$ as $p \rightarrow 0$. Thus $\nu^{\xi} V=\xi_{p}$ and the proof of (2.2) is complete.

Remark. Suppose that $\xi \in \operatorname{Con}(X)$ is minimal; i.e., $\xi$ admits no nontrivial decomposition into a sum of elements of $\operatorname{Exc}(X)$. Then by (2.1) and (2.2) either $\xi \in \operatorname{Inv}(X, M)$ in which case $M=1_{[0, \zeta[}$ a.s. $P^{\xi}$, or $\xi=\nu^{\xi} V \in \operatorname{Pot}(X, M)$. 


\section{Balayage.}

As in previous sections $M$ is an exact MF of $X, S=\inf \left\{t: M_{t}=0\right\}$, and $E_{M}=\{x \in$ $\left.E: P^{x}\left(M_{0}\right)=1\right\}$. However we no longer assume that $S \geq \zeta$ a.s.

For $q \geq 0$ we define

$$
P_{M}^{q} f(x)= \begin{cases}-P^{x} \int_{] 0, \zeta[} e^{-q t} f \circ X_{t} d M_{t}, & x \in E_{M} ; \\ f(x), & x \in E_{M}^{c},\end{cases}
$$

which agrees with (1.2) when $q=0$. Clearly $P_{M}^{q}\left(b \mathcal{E}^{u}\right) \subset b \mathcal{E}^{u}$ and $P_{M}^{q}\left(S^{q}(X)\right) \subset S^{q}(X)$. (Here and in the sequel, if $(F, \mathcal{F})$ is a measurable space, then $\mathcal{F}^{u}$ is the universal completion of $\mathcal{F}$.) As a replacement for $(1.8$ ) (which is valid only when $S \geq \zeta$ ), we have

$$
U^{q}=V^{q}+P_{M}^{q} U^{q}
$$

The operators $P_{M}^{q}$ have duals relative to $L^{q}$ which we now define following $[\mathbf{F G} ; \S 3]$ (where

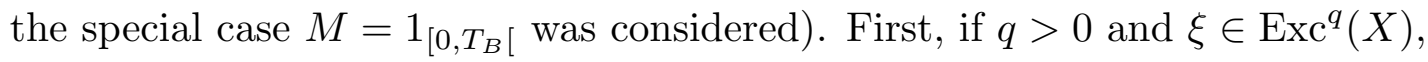

$$
R_{M}^{q} \xi(f):=L^{q}\left(\xi, P_{M}^{q} U^{q} f\right)
$$

where $L^{q}$ is the energy functional of the $q$-subprocess $X^{q}$. As in $[\mathbf{F G}], R_{M}^{q} \xi \in \operatorname{Exc}^{q}(X)$, $R_{M}^{q} \xi \leq \xi$ and if $\mu_{n} U^{q} \uparrow \xi \in \operatorname{Exc}^{q}(X)$ then $R_{M}^{q}\left(\mu_{n} U\right)=\mu_{n} P_{M}^{q} U^{q} \uparrow R_{M}^{q} \xi$. Next, a straightforward computation shows that

$$
(q-r) U^{r} P_{M}^{q} U^{q} \leq P_{M}^{r} U^{r}-P_{M}^{q} U^{q}, \quad 0<r<q
$$

Now if $\xi \in \operatorname{Exc}^{r}(X)$ and $0<r<q$, then we can choose a sequence of measures $\mu_{n}$ such that $\mu_{n} U^{r} \uparrow \xi$; setting $\nu_{n}=\mu_{n}+(q-r) \mu_{n} U^{r}$ we have $\nu_{n} U^{q} \uparrow \xi$, hence by (3.4),

$$
R_{M}^{q} \xi=\lim _{n} \mu_{n}\left(I+(q-r) U^{r}\right) P_{M}^{q} U^{q} \leq \lim _{n} \mu_{n} P_{M}^{r} U^{r}=R_{M}^{r} \xi
$$

Thus $q \mapsto R_{M}^{q} \xi$ is decreasing on $] 0, \infty[$ if $\xi \in \operatorname{Exc}(X)$. We now define

$$
R_{M}^{0} \xi=R_{M} \xi=\uparrow \lim _{q \downarrow 0} R_{M}^{q} \xi, \quad \xi \in \operatorname{Exc}(X)
$$


Evidently $R_{M}: \operatorname{Exc}(X) \rightarrow \operatorname{Exc}(X)$. Various properties of $R_{M}^{q}, q \geq 0$, are established in $[\mathbf{F G} ; \S 3]$ in the special case $M=1_{\left[0, T_{B}[\right.}$. These assertions remain valid here and will be used in the sequel. We mention here a few specifics. First, the relation (3.3) is true when $q=0$ provided $\xi \in \operatorname{Dis}(X)$. Second, the duality formula

$$
L^{q}\left(R_{M}^{q} \xi, u\right)=L^{q}\left(\xi, P_{M}^{q} u\right)
$$

is valid for $q \geq 0, \xi \in \operatorname{Exc}^{q}(X), u \in S^{q}(X)$. Finally, writing $\xi=\xi_{d}+\xi_{c}$ for the decomposition of $\xi \in \operatorname{Exc}(X)$ into dissipative and conservative components, we have $\left(R_{M} \xi\right)_{d}=R_{M}\left(\xi_{d}\right)$ and $\left(R_{M} \xi\right)_{c}=R_{M}\left(\xi_{c}\right)$.

In the remainder of this section we give a precise definition of $\tilde{\xi}$ and $\tilde{u}$ appearing in formula (1.3). In fact we also consider the analogous objects $\tilde{\xi}^{q}$ and $\tilde{u}^{q}, q>0$.

(3.7) PROPOSITION. Fix $q \geq 0$. Given $\xi \in \operatorname{Exc}^{q}(X)$, a $\sigma$-finite measure $\tilde{\xi}^{q}$ on $(E, \mathcal{E})$ is uniquely determined by

$$
\tilde{\xi}^{q}(f)=\xi(f)-R_{M}^{q} \xi(f), \quad f \in p L^{1}(\xi) .
$$

The measure $\tilde{\xi}^{q}$ is carried by $E_{M}$, and if regarded as a measure on $\left(E_{M}, \mathcal{E} \cap E_{M}\right)$ is an element of $\operatorname{Exc}^{q}(X, M)$. If $\xi=\mu U^{q}$, then $\tilde{\xi}^{q}=\mu V^{q}$.

Proof. It suffices to consider the case $q>0$. Then $\tilde{\xi}^{q}\left(E_{M}^{c}\right)=0$ follows easily from (3.2) and (3.3), as does the evaluation $\tilde{\xi}^{q}=\mu V^{q}$ if $\xi=\mu U^{q}$. For a general $\xi \in \operatorname{Exc}^{q}(X)$ (= $\operatorname{Dis}^{q}(X)$ since $q>0$ ), there is a sequence $\left(\mu_{n}\right)$ with $\mu_{n} U^{q} \uparrow \xi$. Then $\mu_{n} P_{M}^{q} U^{q} \uparrow R_{M}^{q} \xi$ as noted earlier; hence by $(3.2), \mu_{n} V^{q} f \rightarrow \tilde{\xi}^{q}(f)$ provided $f \in p L^{1}(\xi)$. Finally, for such $f$,

$$
\tilde{\xi}^{q} Q_{t}^{q} f=\lim _{n} \mu_{n} V^{q} Q_{t}^{q} f \leq \lim _{n} \mu_{n} V^{q} f=\tilde{\xi}^{q}(f),
$$

and so $\tilde{\xi}^{q} \in \operatorname{Exc}^{q}(X, M)$. $\square$ 
The dual definition of $\tilde{u}^{q}$ is a more delicate matter.

(3.8) PROposition. Fix $q \geq 0$. Given $u \in S^{q}(X)$, there exists $\tilde{u}^{q} \in S^{q}(X, M)$ such that

$$
\tilde{u}^{q}=u-P_{M}^{q} u \quad \text { on } \quad\left\{P_{M}^{q} u<\infty\right\} \cap E_{M} .
$$

If $u=U^{q} f$ then $\tilde{u}^{q}=V^{q} f$ on $\left\{P_{M}^{q} u<\infty\right\} \cap E_{M}$.

Proof. Suppose first that $u \in S^{q}(X)$ where $q>0$. Let $B=\left\{P_{M}^{q} u<\infty\right\} \cap E_{M}$. Then $B \in \mathcal{E}^{e}$ is finely open. Since $\left\{P_{M}^{q} u<\infty\right\}$ is absorbing for $X^{q}$, each of the measures $Q_{t}^{q}(x, \cdot), t \geq 0, x \in B$, is carried by $B$. Define $u^{*}$ on $E_{M}$ by

$$
u^{*}(x)= \begin{cases}u(x)-P_{M}^{q} u(x), & x \in B ; \\ \infty, & x \in E_{M} \backslash B .\end{cases}
$$

We claim that $u^{*}$ is $\left(Q_{t}^{q}\right)$-supermedian. Indeed since $q>0$ there are functions $f_{n}$ such that $U^{q} f_{n} \uparrow u$ as $n \rightarrow \infty$. Then $V^{q} f_{n}=U^{q} f_{n}-P_{M}^{q} U^{q} f_{n} \rightarrow u^{*}$ on $B$. Fatou's lemma now shows that $Q_{t}^{q} u^{*} \leq u^{*}, t \geq 0$, as claimed. Now define

$$
\tilde{u}^{q}(x)=\uparrow \lim _{t \downarrow 0} Q_{t}^{q} u^{*}(x), \quad x \in E_{M} .
$$

Clearly $\tilde{u}^{q} \in S^{q}(X, M)$. Writing $u_{n}^{*}=u^{*} \wedge n$ we see that each $u_{n}^{*}$ is $\left(Q_{t}^{q}\right)$-supermedian and finely continuous on $B$. Thus if $x \in B$,

$$
Q_{t}^{q} u_{n}^{*}(x)=P^{x}\left(u_{n}^{*}\left(X_{t}\right) e^{-q t} M_{t}\right) \rightarrow u_{n}^{*}(x), \quad \text { as } \quad t \downarrow 0 .
$$

But $u_{n}^{*} \uparrow u^{*}$ as $n \rightarrow \infty$, and $Q_{t}^{q} u_{n}^{*} \uparrow u_{n}^{*}$ on $B$ as $t \downarrow 0$ because of (3.9). It follows that $\tilde{u}^{q}=u^{*}$ on $B$. In particular $\tilde{u}^{q}=u^{*}=V^{q} f$ on $B$ if $u=U^{q} f$.

It remains to consider the case $q=0$. Fix $u \in S(X) \subset S^{q}(X)$ and define $\tilde{u}^{q}$ as above. Note that $\left\{P_{M} u<\infty\right\} \subset \bigcap_{q>0}\left\{P_{M}^{q}<\infty\right\}$. Thus if $x \in\left\{P_{M} u<\infty\right\} \cap E_{M}$,

$$
u^{*}(x):=u(x)-P_{M} u(x)=u(x)-\lim _{q \downarrow 0} P_{M}^{q} u(x)
$$


(we put $u^{*}=\infty$ on $E_{M} \backslash\left\{P_{M} u<\infty\right\}$ ). Letting $q \downarrow 0$ in the inequality $Q_{t}^{q} \tilde{u}^{q} \leq \tilde{u}^{q}$ we see that $u^{*}$ is $\left(Q_{t}\right)$-supermedian. Setting $\tilde{u}=\uparrow \lim _{t \downarrow 0} Q_{t} u^{*}$, the truncation argument used earlier shows that $\tilde{u}=u^{*}$ on $\left\{P_{M} u<\infty\right\} \cap E_{M}$. Moreover $\tilde{u}=V f$ on $\left\{P_{M} u<\infty\right\} \cap E_{M}$ if $u=U f$

Remark. It is easy to check that the mappings $\xi \mapsto \tilde{\xi}^{q}$ and $u \mapsto \tilde{u}^{q}$ are "positive linear" on their respective domains $\operatorname{Exc}^{q}(X)$ and $S^{q}(X)$.

\section{The Second Formula}

We are now ready to give a precise statement, and proof, of formula (1.3). We shall first state and prove the result for a Borel right process $X$; i.e., $E$ is a Lusin metrizable space with Borel sets $\mathcal{E}$, and each $P_{t}$ maps $b \mathcal{E}$ into itself. This will enable us to use the Kuznetsov process associated with $X$ and $\xi \in \operatorname{Exc}(X)$, and also Meyer's perfection theorem [M74] for $M$. To the best of our knowledge neither the existence of the Kuznetsov process nor Meyer's theorem have been established for general right processes. In (4.19) we shall indicate how formula (3.1) for general right processes may be reduced to the Borel case. As in previous sections $M$ is an exact MF of $X$. We maintain the notation established in previous sections.

(4.1) THEOREM. Assume that $X$ is a Borel right process. Given $\xi \in \operatorname{Exc}(X)$ and $u \in S(X)$,

$$
L(\xi, u)=L^{M}(\tilde{\xi}, \tilde{u})+L\left(\xi, P_{M} u\right)=L^{M}(\tilde{\xi}, \tilde{u})+L\left(R_{M} \xi, u\right)
$$

where $\tilde{\xi} \in \operatorname{Exc}(X, M)$ and $\tilde{u} \in S(X, M)$ are as defined in $\S 3$.

Of course, the second equality in (4.2) expresses the duality between $P_{M}$ and $R_{M}$ already noted. We leave it to the reader to check that these formulas are trivial if either $\xi=\mu U$ or $u=U f$ provided the obvious finiteness conditions are satisfied. However we 
have been unable to use this fact to extend (4.2) to $\xi \in \operatorname{Dis}(X)$ in any straightforward manner. The problem is that $\xi \mapsto \tilde{\xi}$ is not monotone. .

Henceforth we assume that $X$ is a Borel right process; see [G75] or [Sh; (20.6)]. Our notation for the Kuznetsov process $\left(Y, Q_{\xi}\right)$ associated with $X$ and $\xi \in \operatorname{Exc}(X)$ is that used in $[\mathbf{F}]$ and differs slightly from that used in $[\mathbf{F M}]$ or $[\mathbf{G S t}]$. Let $W$ denote the space of paths $w: \mathbb{R} \rightarrow E \cup\{\Delta\}$ that are $E$-valued and right continuous on an open interval $] \alpha(w), \beta(w)[$ and that take the value $\Delta$ elsewhere. The dead path $[\Delta]: t \rightarrow \Delta$ corresponds to $] \alpha, \beta[=\emptyset$, and the appropriate convention is $\alpha([\Delta])=+\infty, \beta([\Delta])=-\infty$. Let $Y=\left(Y_{t}: t \in \mathbb{R}\right)$ denote the coordinate process on $W$, and put $\mathcal{G}^{0}=\sigma\left\{Y_{t}: t \in \mathbb{R}\right\}$ and $\mathcal{G}_{t}^{0}=\sigma\left\{Y_{s}: s \leq t\right\}$.

Given $\xi \in \operatorname{Exc}(X)$, the associated Kuznetsov measure $Q_{\xi}$ is the unique measure on $\left(W, \mathcal{G}^{0}\right)$ not charging $\{[\Delta]\}$ such that if $t_{1}<\cdots<t_{n}$,

$$
\begin{aligned}
Q_{\xi}\left(\alpha<t_{1}, Y_{t_{1}}\right. & \left.\in d x_{1}, \ldots, Y_{t_{n}} \in d x_{n}, t_{n}<\beta\right) \\
& =\xi\left(d x_{1}\right) P_{t_{2}-t_{1}}\left(d x_{2}\right) \cdots P_{t_{n}-t_{n-1}}\left(x_{n-1}, d x_{n}\right) .
\end{aligned}
$$

Evidently $Q_{\xi}$ is $\sigma$-finite and invariant relative to the shift operators $\left(\sigma_{t}\right)$ defined by $Y_{t^{\circ}} \sigma_{s}=$ $Y_{t+s}, s, t \in \mathbb{R}$. Also defined on $W$ are birthing, killing, and shift operators:

$$
\begin{aligned}
&\left(b_{t} w\right)(s)=w(s), \quad s>t \\
&=\Delta, s \leq t ; \\
&\left(k_{t} w\right)(s)=w(s), s<t ; \\
&=\Delta \quad s \geq t . \\
& \theta_{t}=b_{0} \sigma_{t}=\sigma_{t} b_{t}, \quad t \in \mathbb{R} .
\end{aligned}
$$

A convenient way to express the relationship between $X$ and $Y$ is to realize $X$ on the "canonical" space $\Omega=\left\{w \in W: \alpha(w)=0, Y_{\alpha+}(w)\right.$ exists in $\left.E\right\} \cup\{[\Delta]\}$. We let $X_{t}, \mathcal{F}_{t}^{0}$, 
and $\mathcal{F}^{0}$ denote the respective restrictions to $\Omega$ of $Y_{t}, \mathcal{G}_{t}^{0}$ and $\mathcal{G}^{0}$ if $t>0$ and if $t=0$ we let $X_{0}=\left.Y_{0+}\right|_{\Omega}, \mathcal{F}^{0}=\left.\mathcal{G}_{0+}^{0}\right|_{\Omega}$.

Clearly $X_{t+s}=X_{s} \circ \theta_{t}$ if $s, t \geq 0$, while $X_{s} \circ \theta_{t}=Y_{s+t}$ if $s \geq 0, \alpha<t<\infty$. See [FM], $[\mathbf{G S t}],[\mathbf{G 8 8}]$ and $[\mathbf{F}]$ for further details. Since $X$ is Borel, a result of Meyer [M74] allows us to assume that the exact MF $M$ is adapted to $\left(\mathcal{F}_{t+}^{u}\right)$. Following $[\mathbf{G 8 8}]$ we now "extend" $M$ to $W$ : for $\alpha(w)<s \leq t$ define

$$
N(s, t)= \begin{cases}M_{t-s} \circ \theta_{s}, & \alpha<s<t ; \\ 1, & \alpha<s=t .\end{cases}
$$

This makes sense since $\theta_{s}:\{\alpha<s\} \rightarrow \Omega$. The map $s \mapsto N(s, t)$ is increasing and right continuous on $] \alpha, t[$. This allows us to define

$$
N_{t}= \begin{cases}\downarrow \lim _{s \downarrow \alpha} N(s, t), & \alpha<t ; \\ 1, & \alpha \geq t .\end{cases}
$$

Clearly $t \mapsto N_{t}$ is decreasing and right continuous on $] \alpha, \infty\left[, N_{t^{\circ}} \sigma_{s}=N_{t+s}\right.$ for all $s, t \in \mathbb{R}$, and $\left(N_{t}\right)$ is adapted to $\left(\mathcal{G}_{t+}^{u}\right)$. It follows from the multiplicative property of $M$ that $N_{s} N(s, t)=N_{t}$ if $\alpha<s<t$. Letting $s \downarrow \alpha$, then $t \downarrow \alpha$, it follows that $N_{\alpha+}^{2}=N_{\alpha+}$, whence

$$
N_{\alpha+}=0 \quad \text { or } 1 \text {. }
$$

See $[\mathbf{G 8 8}]$ for a complete discussion of these functionals.

The following evaluation is the key to Theorem (4.1).

(4.6) PROPOSITION. If $\xi \in \operatorname{Exc}(X)$, then

$$
R_{M} \xi(f)=Q_{\xi}\left(f \circ Y_{t}\left(1-N_{t}\right)\right), \quad \forall t \in \mathbb{R} .
$$

Remarks. The R.H.S. of (4.7) is independent of $t \in \mathbb{R}$ owing to the $\left(\sigma_{t}\right)$-invariance of $Q_{\xi}$ and the homogeneity of $N_{t}$. In $[\mathbf{F M}]$ the formula (4.7) was taken as the definition 
of $R_{M}$ in the special case $M=1_{\left[0, T_{B}[\right.}$. The identification of this definition with that given in $\S 3$ of this paper was made in $[\mathbf{F G}]$.

Proof of (4.6). First suppose that $\xi \in \operatorname{Dis}(X)$. Define $H$ on $\Omega$ by $H=\int_{0}^{\infty} f \circ X_{t}(1-$ $\left.M_{t}\right) d t$ and note that $H$ is $\mathcal{F}^{u}$-measurable and excessive in the sense that $s \mapsto H \circ \theta_{s}$ is decreasing and right continuous on $\left[0, \infty\left[\right.\right.$. Note that $P^{\cdot}(H)=P_{M} U f$. Define $H^{*}$ on $W$ by

$$
H^{*}=\uparrow \lim _{s \downarrow \alpha} H \circ \theta_{s} .
$$

It is shown in $[\mathbf{F} ;(2.7)]$ that since $\xi \in$ Dis there is a $\mathcal{G}^{0}$-measurable random time $S^{*}: W \rightarrow$ $[-\infty,+\infty]$ such that $t+S^{*} \circ \sigma_{t}=S^{*}$ and $Q_{\xi}\left(S^{*} \notin \mathbb{R}\right)=0$. Moreover, using $[\mathbf{F} ;(4.4)]$,

$$
R_{M} \xi(f)=L\left(\xi, P_{M} U f\right)=Q_{\xi}\left(H^{*} ; 0<S^{*}<1\right)
$$

(The first equality above is just (3.6).) But it is easy to check that

$$
H^{*}=\int_{\mathbb{R}} f \circ Y_{t}\left(1-N_{t}\right) d t=\int d t\left[f \circ Y_{0}\left(1-N_{0}\right)\right] \circ \sigma_{t}
$$

so the third term in (4.8) is precisely $Q_{\xi}\left(f \circ Y_{0}\left(1-N_{0}\right)\right)$ by the "switching identity" (2.1) in $[\mathbf{F}]$. This proves $(4.7)$ in case $\xi \in \operatorname{Dis}(X)$.

To handle $\xi \in \operatorname{Con}(X)$ we use the fact that $\operatorname{Exc}^{q}(X)=\operatorname{Dis}^{q}(X)$ if $q>0$. Fix $\xi \in \operatorname{Con}(X)$ and let $Q_{\xi}^{q}$ be the Kuznetsov process for $X^{q}$ and $\xi$. Then as a special case of the results in [G88], if $F \in p \mathcal{G}^{u}$ with $F([\Delta])=0$,

$$
Q_{\xi}^{q}(F)=Q_{\xi} \iint q^{2} e^{-q(s-r)} 1_{\{r<s\}} F \circ k_{s} \circ b_{r} d r d s .
$$

(One can verify (4.9) directly by checking finite dimensional distributions.) Now since $\xi \in \operatorname{Con}(X) \subset \operatorname{Dis}^{q}(X)$, by what has already been proved,

$$
R_{M}^{q} \xi(f)=Q_{\xi}^{q}\left(f \circ Y_{0}\left(1-N_{0}\right)\right)=Q_{\xi}\left(f \circ Y_{0} \int_{-\infty}^{0} q e^{q r}\left(1-N_{0} \circ b_{r}\right) d r\right),
$$


since $N_{0} \circ k_{s}=N_{0}$ if $\alpha<0<s$. If $r \leq \alpha$ then $N_{0} \circ b_{r}=N_{0}$, while if $\alpha<r<0$ then $N_{0} \circ b_{r}=M_{-r} \circ \theta_{r}$. Therefore

$$
\begin{aligned}
R_{M}^{q} \xi(f)=Q_{\xi}\left(f \circ Y_{0}\left(1-N_{0}\right) e^{q \alpha}\right) & \\
& +Q_{\xi}\left(f \circ Y_{0} \int_{\alpha}^{0} q e^{q r}\left(1-M_{-r} \circ \theta_{r}\right) d r\right) .
\end{aligned}
$$

But $r \mapsto M_{-r} \circ \theta_{r}=N(r, 0)$ is increasing and right continuous on $] \alpha, 0\left[\right.$ with limit $N_{0}$ as $r \downarrow \alpha$. Integrating by parts in (4.10) we obtain

$$
R_{M}^{q} \xi(f)=Q_{\xi}\left(f \circ Y_{0} \int_{] \alpha, 0]} e^{q r} d_{r} N(r, 0)\right) .
$$

Consequently $R_{M} \xi(f)=\uparrow \lim _{q \downarrow 0} R_{M}^{q} \xi(f)=Q_{\xi}\left(f \circ Y_{0}\left(1-N_{0}\right)\right)$, since $N(0,0)=1$. The proof of Proposition (4.6) is complete. $\square$

For the proof of $(4.2)$ in case $\xi \in \operatorname{Con}(X)$ we need the following extension of [GSt; $(6.12)]$.

(4.11) COROLLARY. Define $\varphi=1-P^{\cdot}\left(M_{\zeta_{-}}\right)$. Then $\varphi \in S(X)$, and if $\xi \in \operatorname{Con}(X)$ then $R_{M} \xi=\varphi \cdot \xi$.

Proof. The fact that $\varphi \in S(X)$ is a consequence of the exactness of $M$ which implies that $M_{\zeta-\circ \theta_{t}}$ decreases to $M_{\zeta-}$ as $t$ decreases to 0 . Fix $\xi \in \operatorname{Con}(X)$ so that $Q_{\xi}(\alpha>-\infty)=Q_{\xi}(\beta<+\infty)=0$. Recall that $\left(N_{t}\right)$ is decreasing and homogeneous: $N_{t}^{\circ} \sigma_{s}=N_{t+s}$. Thus if $S_{\lambda}=\inf \left\{t>\alpha: N_{t}<\lambda\right\}$ then $t+S_{\lambda} \circ \sigma_{t}=S_{\lambda}$ for all $t \in \mathbb{R}$. Accordingly, by $[\mathbf{F} ;(2.7)]$, since $\xi \in \operatorname{Con}(X)$ we must have $Q_{\xi}\left(S_{\lambda} \in \mathbb{R}\right)=0$ for all $\lambda \in] 0,1\left[\right.$. But $N_{\alpha+}=0$ or 1 by (4.5); since $t \mapsto N_{t}$ is decreasing and [0,1]-valued, it follows that $N_{t}=N_{\alpha+}$ for all $t>\alpha$, a.s. $Q_{\xi}$. Consider now the "dual" $\left(\hat{N}_{t}\right)$ of $\left(N_{t}\right)$ defined by

$$
\hat{N}_{t}= \begin{cases}\uparrow \lim _{u \uparrow \beta} N(t, u)=M_{\zeta-} \circ \theta_{t}, & \alpha<t<\beta ; \\ 0, & t \leq \alpha ; \\ 1, & t \geq \beta .\end{cases}
$$


Clearly $\left(\hat{N}_{t}\right)$ is increasing and homogeneous. Reasoning as for $\left(N_{t}\right)$ we see that $\hat{N}_{t}=\hat{N}_{\beta-}$ for all $t<\beta$ a.s. $Q_{\xi}$. But $N(s, u)=N(s, t) N(t, u)$ for $\alpha<s<t<u<\beta$, so

$$
N_{\beta-}=N_{t} \hat{N}_{t}=\hat{N}_{\alpha+}, \quad \alpha<t<\beta .
$$

This and the fact that $Q_{\xi}(\alpha>-\infty)=Q_{\xi}(\beta<+\infty)=0$ yields

$$
N . \equiv \hat{N} . \equiv 0 \text { or } 1 \text { a.s. } Q_{\xi} \text {. }
$$

But $\hat{N}_{0}=M_{\zeta-}{ }^{\circ} \theta_{0}$ on $\{\alpha<0<\beta\}$, so using (4.7),

$$
\begin{aligned}
R_{M} \xi(f) & =Q_{\xi}\left(f \circ Y_{0}\left(1-N_{0}\right)\right)=Q_{\xi}\left(f \circ Y_{0}\left(1-\hat{N}_{0}\right)\right) \\
& =Q_{\xi}\left(f \circ Y_{0}\left(1-M_{\zeta-} \circ \theta_{0}\right)\right)=Q_{\xi}\left(f \circ Y_{0} \varphi \circ Y_{0}\right)=\xi(\varphi \cdot f)
\end{aligned}
$$

as claimed.

\section{Proof of (4.2).}

(i) $\xi \in \operatorname{Con}(X)$ : Since $R_{M} \xi \in \operatorname{Con}(X)$ if $\xi \in \operatorname{Con}(X)$, it suffices to show that $\tilde{\xi} \in \operatorname{Con}(X, M)$, for then all terms in $(4.2)$ will vanish. By $(4.11), \tilde{\xi}=(1-\varphi) \cdot \xi$. Since $P^{\xi}(\zeta<\infty)=0$, if we write $M_{\infty}=\lim _{t \uparrow \infty} M_{t}$, then for $t \geq 0$, and $\xi$-a.e. $x \in E$,

$$
\begin{aligned}
\varphi(x) & =P^{x}\left(1-M_{t}\right)+P^{x}\left(M_{t}\left(1-M_{\infty} \circ \theta_{t}\right)\right) \\
& =P^{x}\left(1-M_{t}\right)+P^{x}\left(M_{t} \varphi \circ X_{t}\right)=P^{x}\left(1-M_{t}\right)+\varphi(x) P^{x}\left(M_{t}\right),
\end{aligned}
$$

where the last equality follows since $t \mapsto \varphi \circ X_{t}$ is constant a.s. $P^{\xi}$ (see [GSt; (2.9)]). Letting $t \rightarrow \infty$ above we arrive at $\varphi=\varphi^{2}$ a.e. $\xi$. Thus $\tilde{\xi}=1_{\{\varphi=0\}} \cdot \xi$. Choose $f \in p L^{1}(\xi)$ such that $\{f>0\}=E_{M}$. Then $\xi\{0<U f<\infty\}=0$ since $\xi \in \operatorname{Con}(X)$ (see $[\mathbf{B}]$ or $[\mathbf{D}]$ ). Now $U f=V f+P_{M} U f=V f+\varphi \cdot U f$ a.e. $\xi$ since $t \mapsto U f \circ X_{t}$ is constant a.s. $P^{\xi}$ as noted earlier. Thus $U f=V f$ a.e. $\tilde{\xi}$ and so

$$
\tilde{\xi}(0<V f<\infty)=\tilde{\xi}(0<U f<\infty) \leq \xi(0<U f<\infty)=0 .
$$


Thus $\tilde{\xi} \in \operatorname{Con}(X, M)$ as desired.

(ii) $\xi \in \operatorname{Dis}(X)$ : We first establish (4.2) in the special case $u=1$, in which case $\tilde{1}=1-\varphi=\psi$ (say). Since $\xi \in \operatorname{Dis}(X)$, as noted earlier there is a random time $S^{*} \in \mathcal{G}^{0}$ such that $t+S^{*} \circ \sigma_{t}=S^{*}$ for all $t \in \mathbb{R}$, and $Q_{\xi}\left(S^{*} \notin \mathbb{R}\right)=0$. We shall also need the sequence $\left(S_{n}\right)$ of $\left(\mathcal{G}_{t+}^{0}\right)$-stopping times constructed in [FM; (4.4)] so as to satisfy (a) $t+S_{n^{\circ}} \sigma_{t}=S_{n}$ for all $t \in \mathbb{R}$, (b) $\alpha<S_{n}<\beta$ if $S_{n}<+\infty$, (c) $S_{n} \downarrow \alpha$ as $n \rightarrow \infty$ a.s. $Q_{\xi}$. As noted earlier, $N_{\beta-}=\hat{N}_{\alpha+}=\lim _{t \downarrow \alpha} M_{\zeta-} \circ \theta_{t}$. Thus by [F; (4.4)],

$$
\begin{aligned}
L(\xi, 1) & =Q_{\xi}\left(0<S^{*}<1\right), \\
L\left(\xi, P_{M} 1\right) & =Q_{\xi}\left(1-N_{\beta-} ; 0<S^{*}<1\right) .
\end{aligned}
$$

To prove (4.2) in the present case we must therefore show that

$$
L^{M}(\tilde{\xi}, \tilde{1})=Q_{\xi}\left(N_{\beta-} ; 0<S^{*}<1\right) .
$$

To this end note that $N_{\beta-}$ is $\left(\sigma_{t}\right)$-invariant, so by [F; $\left.(2.4)\right]$ the R.H.S. of (4.12) may be written

$$
\lim _{n \rightarrow \infty} Q_{\xi}\left(N_{\beta-} ; 0<S^{*}<1, S_{n} \in \mathbb{R}\right)=\lim _{n \rightarrow \infty} Q_{\xi}\left(N_{\beta-} ; 0<S_{n}<1\right)
$$

since $Q_{\xi}\left(S^{*} \notin \mathbb{R}\right)=0$. Now $\alpha<S_{n}<\beta$ a.s. $Q_{\xi}$ on $\left\{S_{n}<1\right\}$, so $N_{\beta-}=N_{S_{n}} M_{\zeta-} \circ{ }^{\circ} \theta_{S_{n}}$ a.s. $Q_{\xi}$. The $S_{n}$ being stopping times, we have

$$
Q_{\xi}\left(N_{\beta-} ; 0<S_{n}<1\right)=Q_{\xi}\left(N_{S_{n}} \psi \circ Y_{S_{n}} ; 0<S_{n}<1\right)
$$

by the strong Markov property of $\left(Y_{t}, Q_{\xi}\right)$. (Recall that $\psi=\tilde{1}=P^{\cdot}\left(M_{\zeta_{-}}\right)$.) Now if $t>S_{n}$ then $S_{n} \circ k_{t}=S_{n}$ and $Y_{S_{n}} \circ k_{t}=Y_{S_{n}}$. Also, by the construction of $S_{n}$ in $[\mathbf{F M}]$, $\left\{S_{n} \circ k_{t}=+\infty\right\}=\left\{S_{n} \geq t\right\}$. Since $N_{S_{n}}=-\int_{\left.] S_{n}, \infty\right]} d N_{t}\left(N_{\infty}=0\right)$, using (4.14) we obtain

$$
Q_{\xi}\left(N_{\beta-} ; 0<S_{n}<1\right)=Q_{\xi}\left(-\int_{] \alpha, \infty]}\left(\psi \circ Y_{S_{n}} 1_{] 0,1[}\left(S_{n}\right)\right) \circ k_{t} d N_{t}\right) .
$$


Theorem 4.12(iii) of [ $\mathbf{T}]$ states that his last expression is

$$
Q_{\tilde{\xi}}^{M}\left(\psi \circ Y_{S_{n}} ; 0<S_{n}<1\right)
$$

where $Q_{\tilde{\xi}}^{M}$ is the Kuznetsov measure for $(X, M)$ and $\tilde{\xi} \in \operatorname{Exc}(X, M)$. (This may be verified by a comparison of finite dimensional distributions.) It is easy to check that $S_{n} \downarrow \alpha$ a.s. $Q_{\tilde{\xi}}^{M}$, so applying $[\mathbf{F} ;(2.4)]$ as in $(4.13)$, the expression in (4.15) tends to

$$
L^{M}(\tilde{\xi}, \psi)=L^{M}(\tilde{\xi}, \tilde{1})
$$

as $n \rightarrow \infty$. This combined with (4.12) and (4.13) yields (4.2) for $u=1$.

In proving (4.2) for general $u \in S(X)$ we first consider the case $u<\infty$ a.e. $\xi$. We shall reduce this case to that previously considered by means of the $u$-transform of $X$. We refer the reader to $[\mathbf{G S t}$ for a discussion of the relevant properties of $u$-transforms, and to $[\mathbf{S h} ; \S 62]$ for a complete discussion. According to $[\mathbf{G S h} ;(6.19)]$, given $u \in S(X)$ there is a Borel measurable $\bar{u} \in S(X)$ such that $u=\bar{u}$ off an $M$-polar set. Each of the terms in (4.2) is unchanged if $u$ is replaced by $\bar{u}$, so in the sequel we shall assume without loss of generality that $u$ is Borel. The $u$-transform of $X$ is denoted by $X^{(u)}$ and is the Borel right process on the state space $E_{u}=\{0<u<\infty\}$ with semigroup $P_{t}^{(u)} f=u^{-1} P_{t}(u f)$. In general the superscript $(u)$ will indicate objects defined relative to $X^{(u)}$. (Two exceptions are $P^{x / u}$, the law of $X^{(u)}$ started at $x \in E_{u}$, and $L_{u}$, the energy functional of $X^{(u)}$.)

The following result is well-known for hitting times (and for this result $u$ need not be Borel).

(4.16) PROPOSITION. Fix $u \in S(X)$.

(a) $u \cdot P_{M}^{(u)} 1=P_{M} u$ on $\{u<\infty\}$;

(b) If $\xi \in \operatorname{Exc}(X)$ and $\xi(u=\infty)=0$, then $R_{M}^{(u)}(u \cdot \xi)=u \cdot R_{M} \xi$. 
Proof. In this proof only we write $K^{q}$ for the operator $P_{M}^{q}$ taken relative to $X^{(u)}$ and $W^{q}$ for the resolvent of $X^{(u)}$. As is well-known

$$
u(x) P^{x / u}(F ; t<\zeta)=P^{x}\left(F \cdot u \circ X_{t}\right), \quad F \in b \mathcal{F}_{t+}^{u}
$$

provided $u(x)<\infty$. In this case,

$$
\begin{aligned}
u(x) K^{q} W^{q} f(x) & =u(x) P^{x / u} \int_{0}^{\infty} e^{-q t} f \circ X_{t}\left(1-M_{t}\right) d t \\
& =P_{M}^{q} U^{q}(u f)(x) .
\end{aligned}
$$

If $q>0$ then there are bounded positive $f_{n}$ such that $W^{q} f_{n} \uparrow 1$ on $E_{u}$. But then $U^{q}\left(f_{n} u\right) \uparrow u$ on $\{u<\infty\}$. Moreover, if $u(x)<\infty$ then $P_{M}^{q}(x,\{u=\infty\})=0$. Replacing $f$ by $f_{n}$ in (4.17) and letting $n \rightarrow \infty$ we find that $u \cdot K^{q} 1=P_{M}^{q} u$ on $\{u<\infty\}$. Passing to the limit as $q \downarrow 0$ establishes point (a). With the help of (4.7), point (b) follows exactly as in $[$ GSt; (5.4ii)]. ए

Now fix a (Borel measurable) $u \in S(X)$ with $\xi(u=\infty)=0$. We apply (4.2) with $u=1, X$ replaced by $X^{(u)}$, and $\xi$ replaced by $u \cdot \xi \in \operatorname{Dis}\left(X^{(u)}\right)$ :

$$
\left.L_{u}(u \xi, 1)=\left(L_{u}\right)^{M} \widetilde{u \xi}^{(u)}, \tilde{1}^{(u)}\right)+L_{u}\left(R_{M}^{(u)}(u \xi), 1\right)
$$

But $L_{u}(u \xi, 1)=L(\xi, u)$ by $[\mathbf{G S t} ;(4.10)]$; combining this with (4.16b) shows that the third term in (4.18) reduces to $L\left(R_{M} \xi, u\right)$. It remains to show that the second term in (4.18) equals $L^{M}(\tilde{\xi}, \tilde{u})$. Put $u^{*}=\left.u\right|_{E_{M}}$. Then $u^{*} \in S(X, M)$ and it is easy to check that $\left(X^{(u)}, M\right)=(X, M)^{\left(u^{*}\right)}$, hence $\left(L_{u}\right)^{M}=\left(L^{M}\right)_{u^{*}}$. By $(4.16 \mathrm{~b})$,

$$
\widetilde{u \xi}^{(u)}=u \xi-R_{M}^{(u)}(u \xi)=u \xi-u R_{M} \xi=u \tilde{\xi}=u^{*} \tilde{\xi}
$$

since $\tilde{\xi}$ is carried by $E_{M}$. Also, by (4.16a) and the fact that $P_{M}^{(u)} 1=1$ off $E_{M}^{(u)}=E_{M} \cap E_{u}$,

$$
u^{*} \tilde{1}^{(u)}=u-u P_{M}^{(u)} 1=u-P_{M} u=\tilde{u}
$$


on $E_{M} \cap\{u<\infty\}$, hence a.e. $\tilde{\xi}$. Combining these observations we see that

$$
\left(L_{u}\right)^{M}\left(\widetilde{u \xi}^{(u)}, \tilde{1}^{(u)}\right)=L^{M}(\tilde{\xi}, \tilde{u}),
$$

and (4.2) follows for $\xi \in \operatorname{Dis}(X)$ if $u<\infty$ a.e. $\xi$.

Finally, consider $\xi \in \operatorname{Dis}(X), u \in S(X)$ and suppose that $\xi(u=\infty)>0$. First note that $L(\xi, u)=\infty$. Indeed, choosing $\left(\mu_{n}\right)$ such that $\mu_{n} U \uparrow \xi$, we have $\mu_{n} U(u=\infty)>0$ for all large $n$. But $\{u<\infty\}$ is absorbing so $U(x,\{u=\infty\})=0$ if $u(x)<\infty$. It follows that $\mu_{n}\{u=\infty\}>0$ and that $L(\xi, u)=\uparrow \lim \mu_{n}(u)=\infty$. Thus (4.2) will follow in the present case provided $\xi\left(P_{M} u=\infty\right)>0$. By way of contradiction, assume that $\xi\left(P_{M} u=\infty\right)=0$. Let $B=\{u=\infty\}$. Since $P_{M} u=u$ off $E_{M}, \xi\left(E_{M} \cap B\right)>0$. Clearly $E_{M} \cap\left\{P_{M} u<\infty\right\} \subset\{u<\infty\}^{r}$ (the set of regular points for $\{u<\infty\}$ ), and since $\{u<\infty\}$ is absorbing, $E_{M} \cap\left\{P_{M} u<\infty\right\} \cap B^{r}=\emptyset$. Thus $\xi\left(E_{M} \cap B^{r}\right)=0$. But $B^{r} \subset B$ and $\xi\left(B \backslash B^{r}\right)=0\left(B \backslash B^{r}\right.$ is semipolar). Thus $\xi\left(E_{M} \cap B\right)=0$ and we have our contradiction. The proof of (4.2) is at long last complete.

(4.19) Remarks. We conclude with a brief indication of how Theorem (4.1) may be extended to general right processes. Roughly speaking, given $\xi \in \operatorname{Exc}(X)$, we produce a Borel right process $X^{*}$ with the same finite dimensional distributions as $X$ for $\xi$ a.e. starting point. As far as formula (4.2) is concerned the processes $X$ and $X^{*}$ are "equivalent". Applying Theorem (4.1) to $X^{*}$ we thereby obtain formula (4.2) for $X$.

Passing to the details, let $\left(\bar{X}_{t}, \bar{P}_{t}, \bar{E}\right)$ be a Ray compactification of $X$ as in [Sh; §39]. Fix $\xi \in \operatorname{Exc}(X)$ and choose $E_{0} \in \overline{\mathcal{E}}$ such that $E_{0} \subset E$ and $\xi\left(E \backslash E_{0}\right)=0$. Let $E^{*}=D \cap\left\{x \in \bar{E}: \bar{U}^{1}\left(x, \bar{E} \backslash E_{0}\right)=0\right\}$. Then $\left(E^{*}, \overline{\mathcal{E}} \cap E^{*}\right)$ is a Lusin space, $\xi\left(E \backslash E^{*}\right)=0$, and $E^{*}$ is absorbing for $\bar{X}$. It follows that $X^{*}$, the restriction of $\bar{X}$ to $E^{*}$, is a Borel right process and that $E^{*} \backslash E$ is quasi-polar for $X^{*}$ (see [Sh; (39.15)]). In addition, $E \backslash E^{*}$ is $\xi$-polar for $X$. 
If $\eta \in \operatorname{Exc}(X)$ and $\eta\left(E \backslash E^{*}\right)=0$, then $\eta$ may be regarded as a measure $\eta^{*}$ on $E^{*}$; as such $\eta^{*} \in \operatorname{Exc}\left(X^{*}\right)$. Similarly, if $u \in S(X)$ then $u^{*}(x):=\uparrow \lim _{t \downarrow 0} \bar{P}_{t} u(x), x \in E^{*}$, defines an element $u^{*}$ of $S\left(X^{*}\right)$ such that $u^{*}=u$ on $E \cap E^{*}$. Writing $L^{*}$ for the energy functional of $X^{*}$, we have $L^{*}\left(\eta^{*}, u^{*}\right)=L(\eta, u)$.

Now given an exact MF, $M$, of $X$, there exists an exact MF, $M^{*}$, of $X^{*}$, such that (using the obvious notation)

$$
\left(P_{M} u\right)^{*}=P_{M^{*}}\left(u^{*}\right), \quad\left(R_{M} \eta\right)^{*}=R_{M^{*}}\left(\eta^{*}\right)
$$

provided $u \in S(X), \eta \in \operatorname{Exc}(X)$ with $\eta\left(E \backslash E^{*}\right)=0$. We now apply Theorem (4.1) to $X^{*}$ and the elements $M^{*}, \xi^{*}$, and $u^{*}$, and then verify that each term in (4.2) is unchanged if the $*$ 's are dropped, whence (4.2) for $X, M, \xi$, and $u$. This task is routine, if lengthy, and is left to the interested reader.

\section{References}

[B] Blumenthal, R. M. (1986). A decomposition of excessive measures, in Seminar on Stochastic Processes 1985, pp. 1-8, Birkhäuser, Boston.

[D] Dynkin, E. B. (1980). Minimal excessive measures and functions, Trans. Amer. Math. Soc., 258, 217-244.

[DM] Dellacherie, C., Meyer, P.-A. (1987). Probabilités et Potentiel, Ch. XII à XVI, Hermann, Paris.

[F] Fitzsimmons, P. J. (1988). On a connection between Kuznetsov processes and quasi-processes, in Seminar on Stochastic Processes 1987, pp.123-133, Birkhäuser, Boston.

[FG] Fitzsimmons, P. J., Getoor, R. K. (1988). Revuz measures and time changes. Math. Zeit., 199, 233-256

[FM] Fitzsimmons, P. J. , Maisonneuve, B. (1986). Excessive measures and Markov processes with random birth and death, Probab. Th. Rel. Fields, 72, 319-336.

[G75] Getoor, R. K. (1975). Markov Processes: Ray Processes and Right Processes. Lecture Notes in Math. 440, Springer-Verlag, Berlin-Heidelberg-New York.

[G88] Getoor, R. K. (1988). Killing a Markov process under a stationary measure involves creation, Ann. Probab., 16, 564-585. 
[GSh] Getoor, R. K., Sharpe, M. J. (1984). Naturality, standardness, and weak duality for Markov processes, Z. Warscheinlichkeitstheorie verw. Geb., 67, 1-62.

[GSt] Getoor, R. K. , Steffens J. (1987) The energy functional, balayage, and capacity, Ann. Inst Henri Poincaré, 23, 321-357.

[M66] Meyer,P.-A. (1966). Quelques résultats sur les processus de Markov, Invent. Math. 1, 101-115.

[M74] Meyer, P.-A. (1974). Ensembles aléatoires markoviens homogènes I, in Séminaire de Probabilités VII, Lecture Notes in Math., 321, pp. 176-190, Springer-Verlag, Berlin-Heidelberg-New York.

[Sh] Sharpe, M. J. (1988). General Theory of Markov Processes, Academic Press, New York.

[T] Toby, E. (1988). Birthing and killing a Markov process under a stationary measure, Ph. D. Thesis, University of California, San Diego.

P. J. Fitzsimmons

Department of Mathematics, C-012

University of California, San Diego

La Jolla, California 92093
R. K. Getoor

Department of Mathematics, C-012

University of California, San Diego

La Jolla, California 92093 